\title{
New Applications to Atom Probe Tomography: Insights on Trace Element Diffusion in Naturally Deformed Minerals
}

\author{
R. Dubosq ${ }^{1}$, B. Gault ${ }^{2,3 *}$ A. Rogowitz ${ }^{4}$, K. Schweinar ${ }^{2}$, S. Zaefferer ${ }^{2}$ and D. Schneider ${ }^{1}$ \\ 1. Department of Earth and Environmental Sciences, University of Ottawa, Ottawa, Canada \\ 2. Max-Planck-Institut für Eisenforschung GmbH, Düsseldorf, Germany \\ 3. Department of Materials, Royal School of Mines, Imperial College London, London, UK \\ 4. Department of Geodynamics and Sedimentology, University of Vienna, Vienna, Austria \\ * Corresponding author: b.gault@mpie.de
}

The links between deformation-induced microstructures and trace element diffusion is an important theme within material sciences and geosciences. In material sciences, investigating the interplay between impurities and crystalline defects has had a significant impact on improving the design of synthetic materials $[1,2]$. As for geochronology, the reliable use of minerals such as zircon and titanite as $\mathrm{U}-\mathrm{Th}-\mathrm{Pb}$ geochronometers requires minimal radiogenic isotope and trace element mobility [3, 4]. Deformationinduced redistribution of trace elements within minerals can affect isotopic ratios, significantly impacting the reliability of geochronometers. In economic geology, the incorporation of base- and precious-metals in sulphide minerals has significant implications for metallic ore paragenesis [5]. It has recently been illustrated that plastic deformation in pyrite creates diffusion pathways as subgrain boundaries that act as traps for base- and precious-metals [6]. However, the plastic behaviour of pyrite and the diffusion mechanisms driving trace elements in microstructures remain poorly understood. In this study, we attempt to document the diffusion processes driving trace elements into deformation-induced microstructures of sulphides by developing new applications to 2D and 3D nanostructural and geochemical imaging techniques. Herein, we integrate electron backscatter diffraction (EBSD) mapping, electron channeling contrast imaging (ECCI) and atom probe tomography (APT) on crystal-plastically deformed pyrite. Our study pushes the limits of the 3D tools' abilities by applying new data processing techniques that allow crystallographic-orientation measurement of nanostructural crystal defects.

Preliminary EBSD mapping was conducted on pyrite sites of interest to quantify crystal-plastic deformation. Misorientation deviation angle and grain boundary maps were created using the MTEX toolbox in Matlab [7]. The boundary between two pyrite grains was targeted from the EBSD maps for further analyses. Characterization of deformation-induced microstructures was conducted via ECC imaging [8,9]. Sites of interest within ECC images were selected for APT to interrogate the atomic structure of the deformed zones in an attempt to characterize the trace elements hosted within these features giving insight into the diffusion processes that mobilize them. Specimen reconstruction followed the protocol found in [10], allowing us to manually add the 002 and 111 crystallographic poles in each reconstruction and measure their angles relative to the observed nanostructures. Once those angles were determined the orientation of each structure has been plotted onto a stereographic projection for analysis.

EBSD data exhibit evidence for crystal-plastic deformation within the brittle-dominated pyrite fragments in the form of linear to more heterogeneous misorientation patterns with a maximum misorientation of $10^{\circ}$. Minor development of partial subgrain boundaries $\left(5-10^{\circ}\right)$ occurs at or near the boundary of larger deformed grains (figure 1a). Further ECC images reveal a microfracture at the boundary between two grains in addition to significant evidence of crystal-plastic deformation including higher dislocation density, stacking faults, bend contours, and subgrain boundaries. Specimen reconstructions of this domain based on APT analyses reveals individual linear and planar defects (i.e. dislocations, subgrain boundary, stacking faults; figure 1b) highlighted by elevated As and Co compositions. Compositional profiles across 
one of the documented dislocations reveals a depletion in As directly adjacent to the defect (figure 1c). This observation is consistent with a dislocation-impurity par (DIP) diffusion model where impurities are attracted to dislocation cores by a strain field or 'capture zone' that is created by the dislocation leading to the entrainment of impurities with migrating dislocations $[11,12]$. The absence of such depletion in the wake of other documented dislocations could potentially be due to the onset of volume diffusion. Although we cannot confirm that 'pipe' diffusion also occurs in the investigated sample, we document a subgrain boundary that is enriched in As, therefore it is probable to assume that 'pipe' diffusion could have occurred [13]. In addition, orientation analysis of the documented nanostructures shows that most dislocations and poles to planar structures plot along the 110 planes suggesting possible slip on $\{110\}$.

Our data demonstrates that pyrite deformation in the investigated sample occurred at the brittle-ductile transition zone for pyrite $\left(260-450^{\circ} \mathrm{C}\right)$. Intragrain nanostructures indicative of crystal-plastic deformation include: dislocations, stacking faults, bend contours, and subgrain boundaries. These structures suggest that strain was accommodated by dislocation-glide and -creep with orientation measurements from APT data revealing slip occurred along $\{110\}$ planes. APT analyses also revealed that single isolated dislocations, stacking faults, and subgrain boundaries have elevated compositions of As and Co. Although our APT specimen reconstructions and composition profiles best support a DIP diffusion model for the migration of trace elements during pyrite deformation, we also acknowledge that other diffusion mechanisms including volume and 'pipe' diffusion most likely occurred simultaneously.

\section{References:}

[1] Meng et al, Advanced Energy Materials 7(13) (2017), p. 1-11.

[2] Raabe et al, IOP Conference Series: Materials Science and Engineering 219 (2017), 012006.

[3] Kirkland et al, Chemical geology 483 (2018), p. 558-566.

[4] Piazolo et al, Nature Communications 7 (2016), p. 1-7.

[5] Reich et al, Geochimica et Cosmochimica Acta 69(11) (2005), p. 2781-2796

[6] Dubosq et al, Lithos 310-311 (2018), p. 86-104.

[7] Bachmann et al, Journal of Applied Crystallography 43 (2010), p. 1338-1355.

[8] Zaefferer and NN Elhami, Acta Materialia 75(154) (2014), p. 20-50.

[9] Rogowitz et al, Terra Nova 00 (2018), p. 1-8.

[10] Larson et al in "Local electrode atom probe tomography: A user's guide”, (Springer, Heidelberg).

[11] Cottrell and BA Bilby, Proceedings of the Physical Society A 62(1) (1949), p. 49-62

[12] Petukhov and PA Klyuchnik, Crystallography Reports 57 (2012), p. 388-392.

[13] Love, Acta Metallurgica Materialia 12(6) (1964), p.731-737.
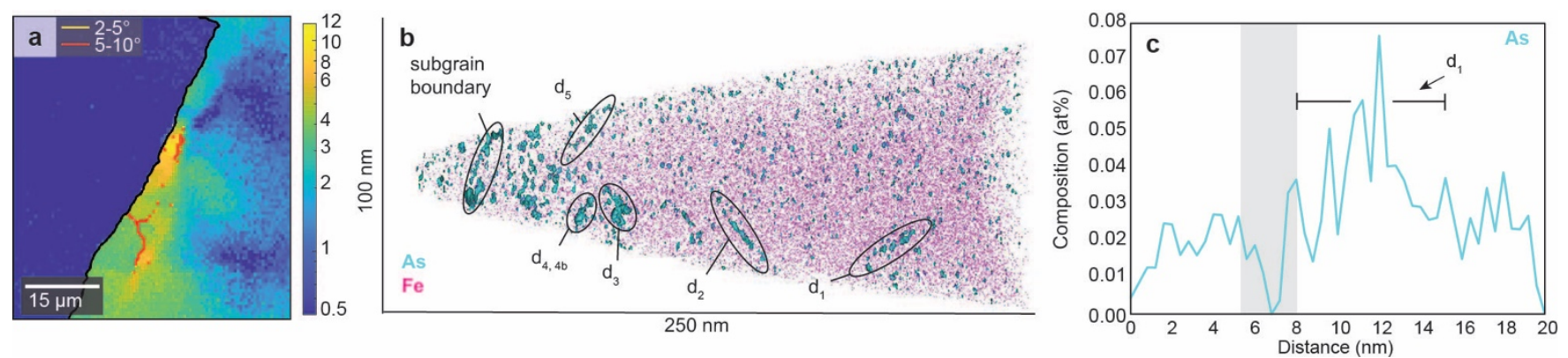

Figure 1. a. EBSD misorientation and grain boundary map of pyrite domain showing maximum misorientation and subgrain boundary development at grain boundary. b. APT specimen reconstruction showing As-rich dislocations $\left(\mathrm{d}_{\mathrm{n}}\right)$ and subgrain boundary. c. Composition profile across dislocation $1\left(\mathrm{~d}_{1}\right)$ showing As depletion in its wake (vertical grey bar). 